\title{
The Synthetic Character Ritchie: First Steps Towards a Virtual Companion for Mixed Reality
}

\author{
Klaus Dorfmüller-Ulhaas and Elisabeth André \\ University of Augsburg, Germany \\ \{dorfmueller-ulhaas,andre\}@informatik.uni-augsburg.de
}

\begin{abstract}
Unlike most existing work on traversable interfaces, we focus on the use of synthetic characters to accompany the user in Mixed Reality (MR) applications. We examine virtual companions as a promising means to design smooth transitions between different worlds and to avoid orientation problems. We propose a taxonomy to describe the spatial relationship between character and user which has an important impact on the style of interaction. To flexibly transfer user and character into different spaces, we have created a platform that supports the design of interfaces derived from the proposed taxonomy as well as transitions between them.
\end{abstract}

\section{Introduction}

Recently, increasing attempts are being made to integrate embodied conversational agents into the user's natural environment $[2,3,1]$. Users and characters no longer inhabit separated spaces, but share an informational and physical reality. Obviously, shared realities bring new perspectives for humans and synthetic individuals to get in touch with each other. A second intriguing development is the realization of so-called traversable interfaces [4] that allow users to cross the border from the digital world to the real world and vice versa. When moving from one space to another, the user has to correctly correlate different visual representations of one and the same object. A basic problem from the perspective of user interface design is therefore the question of how to make the transfer between different worlds as smooth as possible.

We propose the use of a virtual companion that accompanies the user when transferring from one space to the other. First of all, a virtual companion may serve as an anchor and help to avoid orientation problems after a transition. Second, by considering the user's interactions in previously visited spaces, it maintains coherence across spaces. Third, virtual characters allow for communication styles common in human-human dialogue and thus can release users unaccustomed to technology from the burden to learn and familiarize with less native interaction techniques. Last but not least, there is an entertaining value of such a character that makes interactions more enjoyable.

Most virtual character interfaces concentrate on one user-character relation only. Within AR-Quake [5], e.g., user and virtual characters share the real world space. The interactive theatre application [3] and AR Puppet [1] use a movable subspace. Characters are displayed miniaturized. In contrast, we try to use a broader spectrum of coordinate spaces and examine user-character relations.

\section{Coordinate Spaces}

Our platform is based on a short analysis of coordinate spaces synthetic characters can be located in. Figure 1 depicts the relation of a user and a virtual character. User and character either share the same space, or they are separated. Examples are given by the screenshots shown in Fig. 1. User and character can be both located in either a pure virtual (a) or a real environment (b). In addition, we foresee situations in which synthetic characters are located in a separated subspace. In our application, the virtual character can move on top of a real city map (c), or he can appear in the head mounted display the user wears (d). Depending on the choice of user-character relation different methods are required with regard to interaction and conversation. As a result, a traversable interface should provide techniques to store and acquire situation related context information. Thus, we have developed a platform for virtual characters that realizes context switches accross coordinate spaces. Therefore, we distinguish between static and dynamic data. Static data is provided, e.g., by the geometry of the virtual and real world. Most occlusion problems between character and real objects can be solved if a virtual representation of the real environment is available. Dynamic information is updated using sensors in the environment. The basic dynamic data is represented by the current and previous user and character position corresponding to 


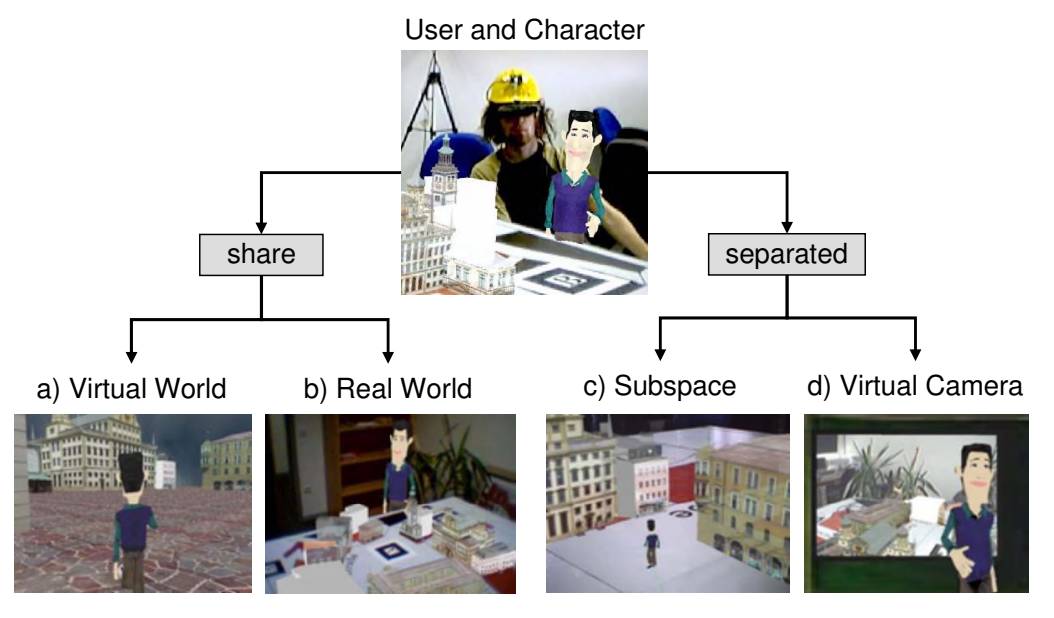

Figure 1. Virtual character interfaces in mixed reality

each coordinate space. This information is necessary when crossing the border from one space to the other.

\section{The Application}

Based on the platform described above, one possible application deploys the synthetic character Ritchie to accompany and guide the user through a virtual 3D environment. A real city map is overlaid by the model of the city center of Augsburg, depending on the user's pose. A frustum of a pyramid with markers attached to each side of it is used for user input (see Fig. 2). The user has the possibility to
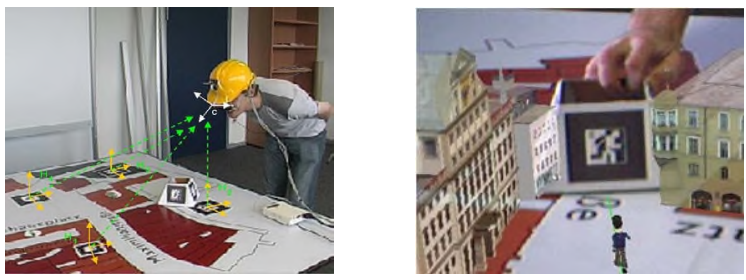

Figure 2. Our table-top application

place the input frustum anywhere on the map while a user selected marker faces towards the camera and triggers a requested action. In our test bed scenario the character Ritchie is located somewhere in the virtual subspace on the real city map (comp. Fig. 1c). After the user has placed the input device on the table, Ritchie searches for the shortest path to the frustum and begins to move in this direction. By watching the character at first, the user is given an impression of the surroundings which is helpful to avoid orientation problems during transition into the virtual world. Briefly after Ritchie started to move, the user is immersed into the virtual world. Now, user and character share the same space. Here, the user has a third person view (comp. Fig. 1a). Our next step will be to examine if Ritchie is actually perceived as a virtual companion.

\section{Current State of the Work}

We have introduced a taxonomy which relates user and character in mixed reality. Furthermore, we have implemented a platform which allows for the flexible configuration of different character-based interfaces for mixed realities. Based on this platform, we are currently preparing a series of user studies with the aim to examine to what extent user engagement is supported by the single modes. In particular, we are interested in the question of how the single modes emphasize the companion role of the character by evoking the feeling of being in the same space with it.

\section{References}

[1] I. Barakonyi, T. Psik, and D. Schmalstieg. Agents that talk and hit back: Animated agents in augmented reality. In IEEE and ACM International Symposium on Mixed and Augmented Reality (ISMAR 2004), pages 141-150, 2004.

[2] M. Cavazza, O. Martin, F. Charles, S.J. Mead, X. Marichal, and A. Nandi. Multi-modal acting in mixed reality interactive storytelling. IEEE Multimedia, 11(3), July-September 2004.

[3] A. D. Cheok, W. Weihua, X. Yang, S. Prince, F. S. Wan, M. Billinghurst, and H. Kato. Interactive theatre experience in embodied + wearable mixed reality space. In ISMAR, pages 59-68, 2002.

[4] B. Koleva, H. Schnädelbach, S. Benford, and C. Greenhalgh. Traversable interfaces between real and virtual worlds. In $\mathrm{CHI}$ '00: Proc. of SIGCHI conference on Human factors in computing systems, pages 233-240, NY, USA, 2000. ACM Press.

[5] W. Piekarski and B. Thomas. Arquake: the outdoor augmented reality gaming system. CACM, 45(1):36-38, 2002. 